\begin{tabular}{|c|c|c|}
\hline$A$ & $\begin{array}{c}\text { International Journal of Current Research } \\
\text { and Academic Review }\end{array}$ & \\
\hline $\begin{array}{l}\text { EXCELLENT } \\
\text { PUBLISHERS }\end{array}$ & 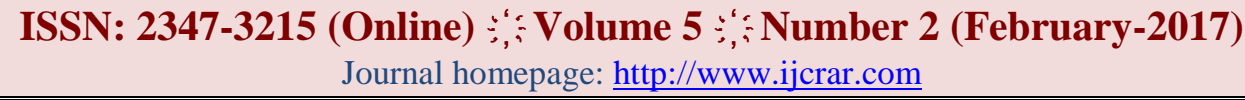 & \\
\hline
\end{tabular}

doi: http://dx.doi.org/10.20546/ijcrar.2017.502.010

\title{
Performance Assessment and Measurements of Mammographic Glandular Dose Using 8000 NERO-mAx and Radcal Acuu-pro X-ray Test Devices
}

\author{
Mohamed Hamed Nassef* \\ Department of Nuclear Engineering, King Abdul-Aziz University, Faculty of Engineering, P.O. Box 80204, Jeddah \\ 21589, Saudi Arabia \\ Nuclear and Radiological Regulatory Authority” (NRRA), Cairo-Egypt*M. H. Nassef (mnassef@kau.edu.sa., on \\ leave from NRRA) \\ *Corresponding author
}

\begin{abstract}
In the present study, a non invasive X-ray test device (8000 Victoreen NERO mAx), and radiation monitor controller (RADCAL-9010) system used to measure the Breast Entrance Dose (BED) in mammographic projection at King Abdulazaiz University Hospital (KAUH), Saudi Arabia. The age distribution and the compressed breast thickness (CBT) of the patients determined based on the information survey from the radiology department logbook. The Average Glandular Dose (AGD) calculated based on the value of the entrance dose and some other imaging technique factors. The X-ray quality control tests were evaluated for beam quality assessment, tube potential accuracy, time accuracy, and reproducibility according to the American College of Radiology (ACR) protocol for the quality control of the mammography imaging technique (Code of practice-TRS 457). The results showed that, good correlation was found between the results obtained from the above mentioned dosemetric systems. The calculated AGD found lower than the international recommended value (3.0 mGy). Also the results give acceptable levels for patient absorbed dose and safety.
\end{abstract}

\section{Article Info}

Accepted: 25 January 2017

Available Online: 20 February 2017

\section{Keywords}

Mammographic entrance dose, Average glandular dose,

Mammography quality control,

Compressed breast thickness.

\section{Introduction}

The most common type of cancer until now is the breast cancer diseases. Breast cancer represents the main disease cause of middle aged women all over the world. It is the highest public concern in the kingdom of Saudi Arabia (KSA). Women breast cancer is the highest type of cancer in the kingdom with a total percentage of 22.4 $\%$. Several studies show that the majority of women breast cancer $(21.6 \%)$ was observed in age group 30-44 years. While the value reach $16.3 \%$ for the age from 45 to 59 years old. Another study in the eastern region of the kingdom show that the highest value for women breast cancer reach $28.7 \%$ which is the highest value till now There are other areas with lower percentage of breast cancer which are the northern part of the kingdom, Makkah, and Qassium regions (Jamal et al., 2011). Recently the mammographic imaging represents the most efficient diagnostic method used to detect any breast cancer with high precision depending on the age of the patient. It was recommended for women to check her breast regularly from any disease. Also it is highly recommend to do this examination yearly after the age of 40 throughout a women's life (Mossang et al., 2011; Assiamah et al., 2005; Dance et al., 1999). Based on the International Commission on Radiological Protection (ICRP) guidelines, the tissue weighting factor for the breast is 0.12 for the effective dose dosimetry (ICRP, 2007; Ferreira et al., 2016). It is essential to optimize the dose transported to the breast to minimize the risk of 
radiation induced cancer. These optimization procedures must cover benefit and risk in other words to increase the benefit and minimize the risk. The improvements on the diagnostic procedure will increase the benefit term in benefit/risk ratio while minimizing the hazard from exposing to the harmful radiation represents the risk factor in that ratio. The actual dose distributed to the breast depends on the physical and geometrical parameters of the mammography unit and on the size of the breast/phantom composition (Dance at al., 1999; EC, 1996). The AGD is the physical quantity dose index that constitutes the average radiation dose to the breast. This is because the breast glandular tissues are more radiosensitive than adipose tissue. The AGD is the most measure of radiation risk arises from the mammography unit and currently accepted descriptor to the breast dose. By measuring the entrance surface exposure to the breast, the AGD can be estimated through the conversion of the entrance exposure dose and applying some conversion factors. The entrance surface exposure can be determined directly by measuring the output of the X-ray tube output using mammographic flat ion chamber (Ki et al., 2003; Assiamah et al., 2005; Florian et al., 2009). The quality control program is useful to produce high mammographic image quality with high resolution to show the anatomy of the breast especially its details and able to detect and identify any disease signs before patient's care is affected. Any small variation or change in the performance of the screening unit or in the film processing system (conventional/digital) can affect the image quality and of course on the value of the dose delivered to the breast. Based on ACR protocol the phantoms are important tools for achieving the quality control procedures for any mammographic unit and to help in the evaluation of the image and accurate characterization of patient breast dose (ACR, 1999; Mitsuhiro et al., 2003; Yanpeng et al., 2010). In this study, the breast entrance dose and the AGD were determined using two dosimetry systems (8000 NERO $\mathrm{mAx}$ and RADCAL-9010) for comparison. A mammographic slab phantom with different thickness was used in this study. The ACR quality control protocol for the technical and physical aspects of mammographic imaging technique was applied.

\section{Materials and Methods}

In this study, a GE Senograph 2000 D, manufactured at USA mammography X-ray machine at King King Abdulaziz University Hospital (KAUH) was used. The mammography machine use $\mathrm{M}_{\mathrm{o}}$ anode plus molybdenum filter. The specification of the used machine was shown in table 1 . The clinical radiographic exposure parameters (tube potential, tube loading, exposure time), patient information including: age, projection type, and CBT were collected and recorded. The non invasive X-ray test device 8000 Victoreen NERO mAx instrument was used with a mammographic ionization chamber Model (Victoreen 6000-529, Fluke, Cleveland, OH). The ion chamber was connected to a calibrated electronic reader. The radiation monitor controller Model (RADCAL9010) device connected with a dedicated mammographic chamber Model 10×6-6 was also used for comparison. Both systems were used to measure the physical parameters of the mammographic machine, the radiation output ( $\mathrm{mGy} / \mathrm{mAs}$ ), and the breast entrance dose. The ionization chamber was fixed at the center of the field of the radiation beam provided that the center of the chamber was positioned at $4 \mathrm{~cm}$ from the chest-wall edge of the image receptor. The patient exposures were done in automatic exposure control system (AEC), i.e. the automatic choice of tube potential and tube current loading. The beam quality was measured by examining the half value layer (HVL) thickness at $28 \mathrm{kVp}$ using high purity aluminum sheets of $99.9 \%$ purity. It is important to mention that, all projections done at a $\mathrm{kVp}$ ranged from $27-28 \mathrm{kVp}, 100-120 \mathrm{mAs}$, with maximum field size $(19 \times 23) \mathrm{cm}^{2}$, and $60 \mathrm{~cm}$ source-to-image distance (SID).

A slab tissue equivalent phantom with different thickness was used to study the breast dose without interference with patient examinations. The thickness of the phantom used in this study was chosen to be $4.5 \mathrm{~cm}$ based on the average registered clinical value of the CBT for all the patients under investigation at KAUH. It is important to mention that, the slab phantom helps us to take more reproducible results based on the size, shape and the ratio of mammary glands to fat tissues of the breast. The test device 8000 Victoreen NERO $\mathrm{mAx}$ measures the average tube potential, the output exposure in unit of $\mathrm{mGy}$ or $\mathrm{mR}$, and the exposure time in (msec), in addition to the half value layer (HVL) measurements. It was attached with an external calibrated mammographic ion chamber $3.3 \mathrm{cc}$ Victoreen Model 6000-529 with a sensitivity of $1 \mathrm{nC} / \mathrm{R}$ and energy response within $5 \%$. The radiation monitor controller (RADCAL corporation model 9010) measure the output exposure in mGy, the exposure time in msec, the tube potential and was attached with external flat ion chamber Model 10x6-6 with energy response over $10-40 \mathrm{keV}$ and the pulse width extends from $10 \mathrm{~ms}$ to $9999 \mathrm{~s}$. The flat chamber is a parallel plate type with a flat response to mammographic examinations, where its wall is made of graphite-coated acrylic. The ion chamber was positioned 
at the $4 \mathrm{~cm}$ reference point $(4 \mathrm{~cm})$. The compression paddle was placed on the top of the chamber to avoid all backscattering effects. Before measuring breast entrance dose, the quality control program of the machine was checked and assessed. The image quality was assessed by using performance breast phantom. The entrance exposure was converted to the average glandular dose (AGD) according to the used protocol (Perry et al., 2006; IAEA, 2007, Marianne et al., 2008; Baoying at al., 2012). Patients average glandular dose per exposure were calculated according to equation 1 . The average glandular tissue dose varies with the incident exposure at the surface of the breast and $4.5 \mathrm{~cm}$ CBT.

Assuming that, the composition is $50 \%$ glandular tissue and $50 \%$ adipose tissue.

$\mathrm{AGD}=\mathrm{K} \times \mathrm{c} \times \mathrm{g} \times \mathrm{s}(1)$

Where the factor c converts air kerma for the Perspex phantom to that for the standard breast (granularity of the tissue) and the factor $g$ converts air kerma for the standard breast to the mean glandular tissue dose for Xray spectra obtained from molybdenum target used a molybdenum filter characterized by the half-value layer in aluminum. The factor s corrects for X-ray spectral differences arising from the use of alternative target/filter combinations (Dance et al., 1999; Boone, 1999, Mariana et al., 2015). The assumption of $50 \%$ granularity is approximately correct for breast thickness of $4-6 \mathrm{~cm}$. The mammography ionization chamber was positioned at the same level $(4.5 \mathrm{~cm})$ as for the surface of the phantom above the breast support and $4 \mathrm{~cm}$ from the chest wall edge. The tube potential used in this study ranged from $28-30 \mathrm{kVp}$ (same clinical condition), with maximum field size of $18 \times 24 \mathrm{~cm}^{2}$.

\section{Results and Discussion}

The mammographic exposure parameters and patient information's were collected from the patient information logbook at KAUH radiology department, mammography section during the period from December 2009 to March 2010. Figure 1 shows the results of the age distribution of the patient's taken from the clinical records logbook files. It was found that, about $22 \%$ of the patients were located within the age interval from 46 to 50 years, $21 \%$ within the age interval from 51 to 55 years and $18 \%$ within the age interval from 41 to 45 years. The other interval was found with low percentage. Approximately, the majority of the patient ages at KAUH are within the interval from 41 to 55 years old. The obtained survey show good agreements and matching with a previous data obtained for different authors (Jamal et al., 2011; Alice, 2012; Soha, 2007).

\section{Performance assessment}

The accuracy of tube voltage in the range from 25-29 $\mathrm{kVp}$ was acceptable as shown in table 2. It was found that the measured tube potential has an acceptable error from the nominal value by about $2.8 \%$ for NERO system and $4.6 \%$ for RADCAL system. Both values are accepted but NERO system show more stability in the higher station for tube potential.

The variation in mammographic tube output $(\mathrm{mR} / \mathrm{mAs})$ versus the nominal tube potential $(\mathrm{kVp})$ is shown in table 3 . The measured value of the tube output increases with increasing the tube potential for the same mammographic machine.

The exposure linearity was measured in which the value of $\mathrm{mR} / \mathrm{mAs}$ is determined at constant tube potential $(\mathrm{kVp}=29)$. The ratio $\mathrm{mR} / \mathrm{mAs}$ were determined at different value of $\mathrm{mAs}$ and were found to be constant as shown in table 4.

\section{Beam quality (HVL) measurements}

Based on the ACR protocol the measurement of the HVL values was discussed as follow: the exposure was taken by the detector at clinical exposure factors without adding any aluminum sheet. Then, by adding aluminum sheet starting from $0.1 \mathrm{~mm} \mathrm{Al}$ and fixed on the top of the compression paddle then another exposure was done to record a new reading and the results were recorded and registered. The previous step was repeated by increasing different aluminum thickness till $0.6 \mathrm{~mm} \mathrm{Al}$ (high purity $99.0 \%$ ) until the new reading reduced in its value and become less than one-half of the original reading. The reading can be plotted on a graph scale or in a personal computer program to determine or to calculate the HVL with a logarithmic scale interpolation analysis. In our study, the HVL was checked at clinically used tube potential $(28 \mathrm{kVp})$ using 8000 NERO mAx non invasive $\mathrm{X}$-ray test device. table 5 and figure 2 show the results of the measured HVL at the following clinical exposure factors $\left(28 \mathrm{kVp}, 30 \mathrm{~cm} \mathrm{SID}, 100 \mathrm{mAs}\right.$, and $9 \times 9 \mathrm{~cm}^{2}$ Field Size).

Figure 2 explains the relation between the tube output exposure against the thickness of the added aluminum filter. The estimated HVL value was found to be 0.32 
$\mathrm{mm} \mathrm{Al}$ and within the recommended value for $\mathrm{M}_{\mathrm{O}} / \mathrm{M}_{\mathrm{O}}$ Target /Filter which ranged from 0.31 to $0.38 \mathrm{~mm} \mathrm{Al}$ i.e. $0.31<\mathrm{HVL}<0.38 \mathrm{~mm} \mathrm{Al}$ at $28 \mathrm{kVp}$ (Mossang et al., 2011, Hendrick, 1999).

\section{Breast entrance surface exposure dose (mGy) measurements}

The breast entrance exposure was measured using the two instruments mentioned above. Table 6 shows the measured dose at $28 \mathrm{kVp}, 100 \mathrm{mAs}, 60 \mathrm{~cm} \mathrm{SID,} 19 \times 23$ $\mathrm{cm}^{2}$ field size, and $4.5 \mathrm{~cm}$ compressed breast thickness (CBT) for off-axis exposure geometry based on ACR protocol. So the inoization chamber was positoioned beside the phantom (breast-equivalent material) at the same hight.

Repeated measurements (six) for the entrance surface exposure show differences between the readings of the two systems. The average value of the dose measured by RADCAL system is $11.39 \mathrm{mGy}$ and the corresponding value for NERO system is $9.64 \mathrm{mGy}$ with a percentage difference of $15 \%$. The difference between the two readings may be due to the main characteristic of the system and the sensitivity of its electronic accessories such as the used ion chamber. However, the NERO-8000 shows good stability in its reading with coefficient of variation of $0.04 \%$.

Table.1 Specification of the used mammographic X-ray machine (physical and geometrical) parameters at KAUH

\begin{tabular}{|l|c|}
\hline Type of generator & GE Senograph 2000D \\
\hline Range of Tube Potential kVp for CC or MLO Projection & $27-28$ \\
\hline Range of Tube Exposure mAs & $100-120$ \\
\hline Focus-to-Film Distance (FFD) & $650 \mathrm{~mm}$ \\
\hline Compressed Breast Thickness (CBT) & $4.5 \mathrm{~mm}$ \\
\hline Field Size & $19 \times 23 \mathrm{~cm}^{2}$ \\
\hline Target / Filter Combination & $\mathrm{Mo} / \mathrm{Mo}$ \\
\hline Focal Spot & $0.15-0.3 / 0.15-0.3 \mathrm{~mm}$ \\
\hline Inherent Filtration & $0.69 \mathrm{~mm} \mathrm{Be}$ \\
\hline
\end{tabular}

Table.2 Set and measured tube potential with the percentage of the bias using two instruments (NERO and RADCAL)

\begin{tabular}{|c|c|c|c|c|}
\hline \multirow{2}{*}{$\begin{array}{c}\text { Set tube potential } \\
(\mathrm{kVp})\end{array}$} & \multicolumn{4}{|c|}{ Xeasured tube potential (Average value) } \\
\cline { 2 - 5 } & \multicolumn{4}{|c|}{ X-ray Test Device } \\
\cline { 2 - 5 } & RADCAL-9010 & \% Bias & 8000 NERO mAx & $\%$ Bias \\
\hline 25 & 24.8 & 0.8 & 25.7 & 2.8 \\
\hline 26 & 25.9 & 0.4 & 26.5 & 1.9 \\
\hline 27 & 26.6 & 1.5 & 27.5 & 1.9 \\
\hline 28 & 26.7 & 4.6 & 28.5 & 1.8 \\
\hline 29 & 28.2 & 2.8 & 29.6 & 2.1 \\
\hline
\end{tabular}

Table.3 The measured values of the output exposure at different values of tube potential

\begin{tabular}{|c|c|c|}
\hline Set $\mathrm{kVp}$ & Measured output $(\mathrm{mR})$ & $\mathrm{mR} / \mathrm{mAs}$ \\
\hline 25 & 743.5 & 7.44 \\
\hline 26 & 854.3 & 8.54 \\
\hline 27 & 973.8 & 9.74 \\
\hline 28 & 1099 & 10.99 \\
\hline 29 & 1231 & 12.31 \\
\hline
\end{tabular}

$\mathrm{SID}=60 \mathrm{~cm}, \mathrm{mAs}=100$ 
Table.4 The variation in output of X-ray tube at constant tube potential

\begin{tabular}{|c|c|c|}
\hline Nominal mAs & Measured output $(\mathrm{mR})$ & $\mathrm{mR} / \mathrm{mAs}$ \\
\hline 63 & 772.9 & 12.26 \\
\hline 71 & 871.9 & 12.28 \\
\hline 80 & 982.9 & 12.29 \\
\hline 100 & 1231 & 12.31 \\
\hline 110 & 1354 & 13.54 \\
\hline
\end{tabular}

$\mathrm{SID}=60 \mathrm{~cm}, \mathrm{kVp}=29,19 \times 23 \mathrm{~cm}^{2}, \mathrm{CV}=4 \%$.

Table.5 The measured values of the HVL at different thicknesses from aluminum attenuators

\begin{tabular}{|c|c|c|c|c|c|}
\hline $\begin{array}{c}\text { Reading } \\
\text { No. }\end{array}$ & $\mathrm{kVp}$ Avg. & $\begin{array}{c}\text { Measured } \\
\text { time msec }\end{array}$ & $\begin{array}{c}\text { Attenuator } \\
\text { thickness } \\
(\mathrm{mm} \mathrm{Al})\end{array}$ & $\begin{array}{c}\text { output Exposure } \\
(\mathrm{mR})\end{array}$ & $\begin{array}{c}\text { Normal } \\
\text { Exposure }\end{array}$ \\
\hline 1 & 28.2 & 1.053 & 0 & 1102 & 1.0 \\
\hline 2 & 28.4 & 1.053 & 0.1 & 891.8 & 0.81 \\
\hline 3 & 28.7 & 1.053 & 0.2 & 732.1 & 0.66 \\
\hline 4 & 29.0 & 1.053 & 0.3 & 608.9 & 0.55 \\
\hline 5 & 29.2 & 1.053 & 0.4 & 508.2 & 0.46 \\
\hline 6 & 28.5 & 1.052 & 0.5 & 429.1 & 0.39 \\
\hline 7 & 29.7 & 1.052 & 0.6 & 383.7 & 0.35 \\
\hline
\end{tabular}

Table.6 Measured breast entrance dose (mGy)

\begin{tabular}{|c|c|c|}
\hline No. & RADCAL-9010 system & 8000 NERO mAx system \\
\hline 1 & 11.38 & 9.65 \\
\hline 2 & 11.38 & 9.64 \\
\hline 3 & 11.39 & 9.64 \\
\hline 4 & 11.38 & 9.64 \\
\hline 5 & 11.39 & 9.64 \\
\hline 6 & 11.39 & 9.64 \\
\hline
\end{tabular}

Table.7 Calculated average glandular dose (AGD) in mGy

\begin{tabular}{|c|c|c|}
\hline No. & (RADCAL-9010) & (8000 NERO-mAx) \\
\hline 1 & 2.23 & 2.21 \\
\hline 2 & 2.23 & 2.21 \\
\hline 3 & 2.23 & 2.21 \\
\hline 4 & 2.23 & 2.21 \\
\hline 5 & 2.23 & 2.21 \\
\hline 6 & 2.23 & 2.21 \\
\hline
\end{tabular}


Fig.1 Pie chart for the age distribution for women patients at KAUH mammography projection

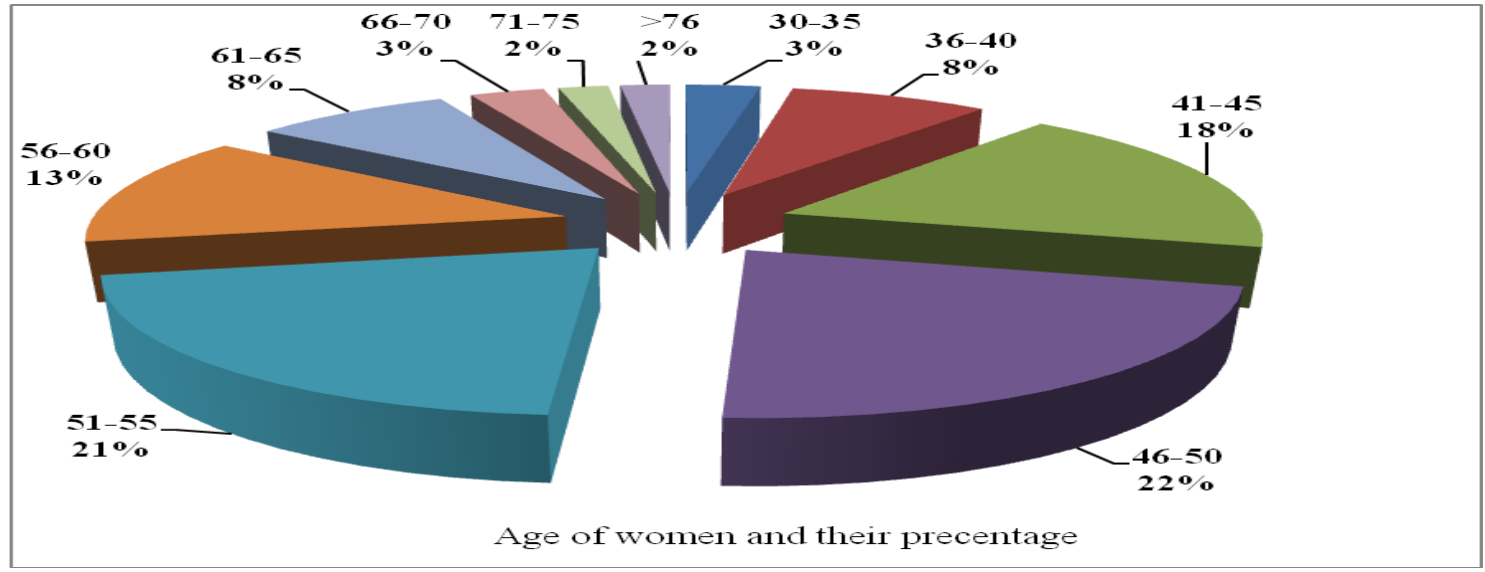

Fig.2 The measured values of the HVL at different thicknesses from $\mathrm{mm} \mathrm{Al}$ attenuators

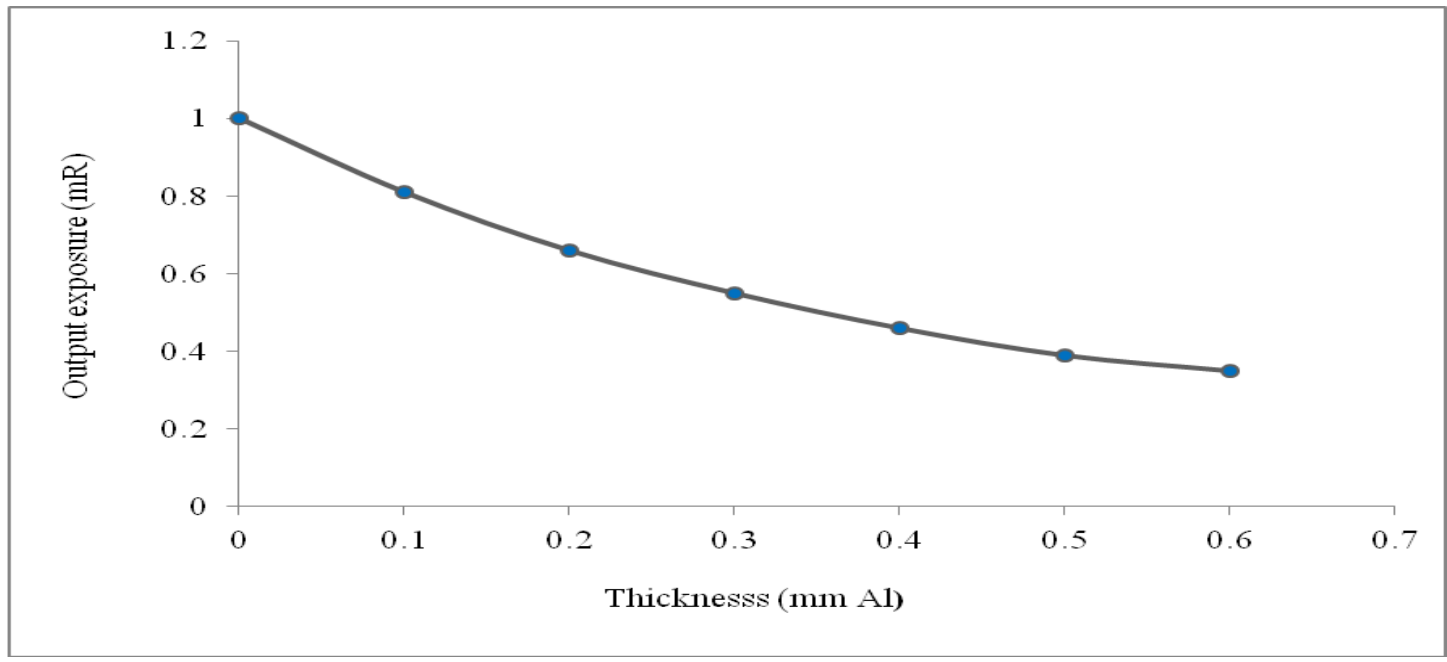

Fig.3 Histogram of the average glandular dose measured by RADCAL 9010 and NERO mAx system

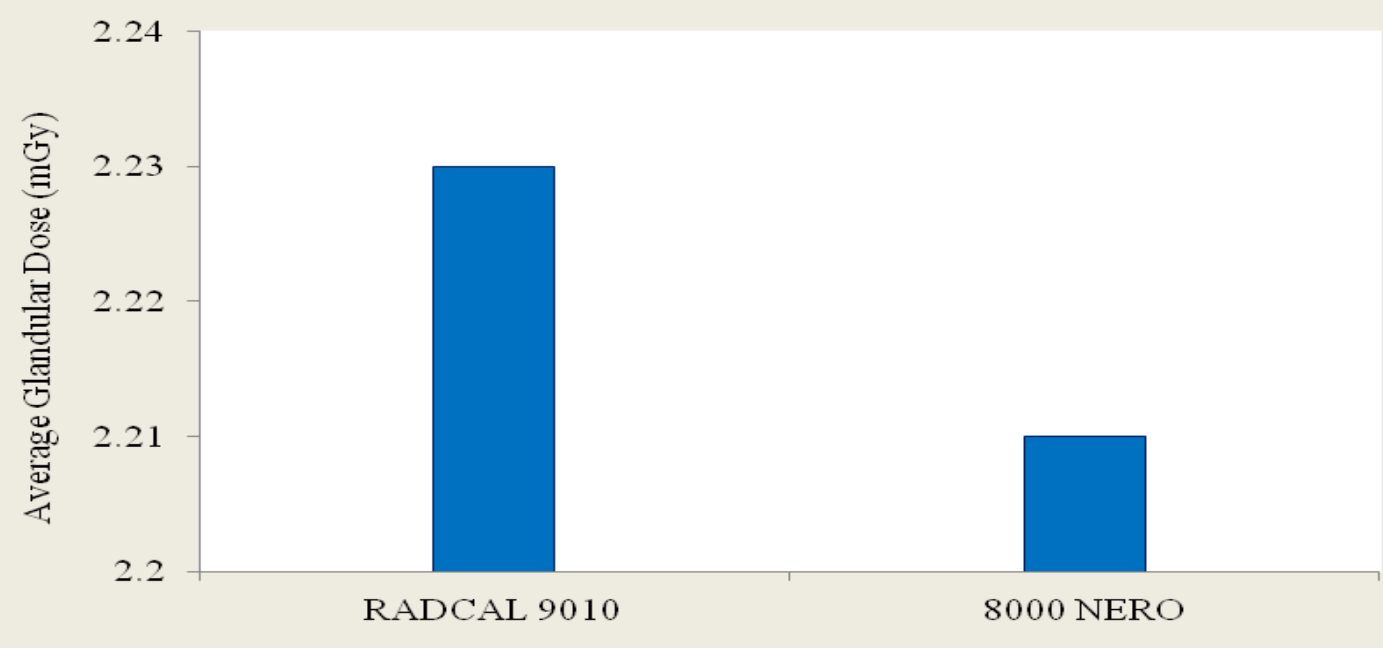




\section{Average glandular dose calculation}

The average glandular dose was determined by applying equation 1 and some conversion factors according to the applied protocol based on the breast compressed thickness, the value of the factors $\mathrm{g}$, c, and s was selected in addition to the determined value of the HVL was introduced in equation 1 to calculate the average glandular dose. Table 7 shows the value of the AGD in mGy based on RADCAL-9010 and 8000 NERO-mAx measurements taking in consideration the value of the following factors $(\mathrm{g}=0.177, \mathrm{c}=1.105, \mathrm{~s}=1)$. These data was presented in figure 3 . The calculated average glandular dose does not exceed the recommended international value $(3 \mathrm{mGy})$. There is a good linearity between the readings for both instruments and the mean value of the glandular dose and it seem to be around 2.2 mGy.

\section{Conclusion}

The average MGD per film was found to be $2.2 \mathrm{mGy}$ in the present study which is lower than the international reference value $(3.0 \mathrm{mGy})$. This indicate that the mammography machine at KSAH capable of achieving acceptable dose levels for all mammographic projections such as Cranial-Caudal view and The Mediolateral Oblique view. A quality control protocol is important and necessary to achieve an optimum mammographic image quality which enables us to evaluate correctly the function of the mammographic machine. The used instruments show good agreement and satisfactory in measuring the tube potential, exposure time, tube output exposure and the absorbed dose delivered to the breast.

\section{Acknowledgement}

The author expresses his high consideration for fruitful cooperation received from both the Heads of Diagnostic Radiology Department and the Mammography Technicians at KAUH.

\section{References}

Assiamah, M., Nam, T.L., and Keddy, R.J. 2005. Comparison of mammography radiation dose values obtained from direct incident air kerma measurements with values from measured X-ray spectral data. Applied radiation and isotopes. 62: 551-560.
American College of Radiology (ACR).1999. Mammography quality control manual, Washington, DC: U.S. Government Relations Office.

Alice, Franklin., 2012. Mammography Screening Efficacy for Breast Cancer Detection in Saudi Arabia. Austral-Asian Journal of Cancer. 11 (3): 213-215.

Baoying Chen., Yingmei Wang., Xin Sun., Wei Guo., Ming Zhao., Guangbin Cui., Lina Hu., Pei Li., Yan Ren., Jun Feng., and Jun Yu. 2012. Analysis of patient dose in full field digital mammography. European Journal of Radiology. 81: 868-872.

Boone, j., 1999. Glandular breast dose for monoenergetic and high-energy $\mathrm{x}$-ray beams: Monte Carlo assessment. Radiology. 213: 23-37.

Dance D.R., Skinner C.L., Alm C.G. 1999. Breast dosimetry. Applied Radiation and Isotopes. 50:185203.

European Commission (EC), 1996. European guidelines on quality criteria for diagnostic radiographic images, EC, Brussels, Office for Official Publications of the European Communities EUR 16260.

Ferreira P., Baptista M., Di Maria S., and Vaz P. 2016. Cancer risk estimation in Digital Breast Tomosynthesis using GEANT4 Monte Carlo simulations and voxel phantoms. Physica Medica. 32: 717-723.

Florian JF Engel., MBChB., Henning Meyer., Ralf Juran., Ulrich Bick., Eva Fallenberg., and Felix Diekmann. 2009. Intra-individual Comparison of Average Glandular Dose of Two Digital Mammography Units using Different Anode/Filter Combinations. Academic Radiology.16 (10): 12721280.

Hendrick RE, 1999. Mammography Quality Control Manual: Medical Physicist's Section. In: Mammography Quality Control Manual 1999. American College of Radiology. 225-330.

International Atomic Energy Agency (IAEA), 2007. Dosimetry in diagnostic radiology: an international code of practice, Technical Reports Series, Vienna, No. 457.

International Commission on Radiological Protection (ICRP), 2007. Recommendations of the ICRP, ICRP Publication 103 (user's edition), Ann. ICRP 37(2-4).

Jamal M., Ahmad M., Mohammed A., and Othman A.2011. Causative relationship between Diabetes Mellitus and breast cancer in various regions of Saudi Arabia: An Overview. Asian Pacific Journal of cancer prevention. 12: 589-592. 
Ki Keun Oh., Jin Hur., Eun-Kyung Kim., and Sung Sil Choo. 2003. Dosimetric evaluation of the mean glandular dos for mammography in Korean women: A preliminary report. Yonsi Medical Journal. 44(5):863-868.

Mariana B., Salvatore Di M., and Silvia B. 2015. Dosimetric characterization and organ dose assessment in digital breast tomosynthesis: Measurements and Monte Carlo simulations using voxel phantoms. Med. Phys. 42 (7): 3788-3800.

Marianne Aznar., and Bengt Hemdal. 2008. Absorbed Dose Measurement in Mammography. Cancer Imaging. 1:493-501.

Mitsuhiro Matsumoto., Shinichi Inoue., Ikuko Honda., Shuji Yamamoto., Takashi Ueguchi., Yuji Ogata., and Takeshi Johkoh. 2003. Real-time estimation system for mean glandular dose in mammography. Radiation Medicine. 21 (6): 280-284.
Mossang D., and Dadulescu E. 2011. Assessing image quality and estimating the glandular average dose in some mammography Lab"s. Romanian Reports in Physics. 63(1): 95-106.

Perry N., Broeders M., de Wolf C., Tornberg S., Holland R., von Karsa L., and Puthaar E. 2006. European guidelines for quality assurance in breast cancer screening and diagnosis. Fourth Edition.

Yanpeng Li., Ann Poulos., Donald McLeana., and Mary Rickarda. 2010. A review of methods of clinical image quality evaluation in mammography. European Journal of Radiology. 74: 122-131.

Soha S. El Shreef, 2007. Breast cancer in Jeddah province: study of area geographic. Master Thesis, Geographic department, Faculty of arts and human science, KAU University.

\section{How to cite this article:}

Mohamed Hamed Nassef. 2017. Performance Assessment and Measurements of Mammographic Glandular Dose Using 8000 NERO-mAx and Radcal Acuu-pro X-ray Test Devices. Int.J.Curr.Res.Aca.Rev. 5(2), 71-78. doi: http://dx.doi.org/10.20546/ijcrar.2017.502.010 